\title{
Functional prediction of differentially expressed IncRNAs in HSV-1 infected human foreskin fibroblasts
}

\author{
Benxia Hu ${ }^{1,2}$, Yongxia Huo ${ }^{1}$, Guijun Chen', Liping Yang ${ }^{1}$, Dongdong $\mathrm{Wu}^{3}$ and Jumin Zhou ${ }^{*}$
}

\begin{abstract}
Background: One of the most important functions of long noncoding RNAs (IncRNAs) is to control protein coding gene transcription by acting locally in cis, or remotely in trans. Herpes Simplex Virus type I (HSV-1) latently infects over $80 \%$ of the population, its reactivation from latency usually results in productive infections in human epithelial cells, and is responsible for the common cold sores and genital Herpes. HSV-1 productive infection leads to profound changes in the host cells, including the host transcriptome. However, how genome wide IncRNAs expressions are affected by the infection and how IncRNAs expression relates to protein coding gene expression have not been analyzed.

Methods: We analyzed differentially expressed IncRNAs and their potential targets from RNA-seq data in HSV-1 infected human foreskin fibroblast (HFF) cells. Based on correlations of expression patterns of differentially expressed protein-coding genes and IncRNAs, we predicted that these IncRNAs may regulate, either in cis or in trans, the expression of many cellular protein-coding genes.

Results: Here we analyzed HSV-1 infection induced, differentially expressed IncRNAs and predicted their target genes. We detected 208 annotated and 206 novel differentially expressed IncRNAs. Gene Ontology and Pathway enrichment analyses revealed potential IncRNA targets, including genes in chromatin assembly, genes in neuronal development and neurodegenerative diseases and genes in the immune response, such as Toll-like receptor signaling and RIG-I-like receptor signaling pathways.

Conclusions: We found that differentially expressed IncRNAs may regulate the expression of many cellular proteincoding genes involved in pathways from native immunity to neuronal development, thus revealing important roles of IncRNAs in the regulation of host transcriptional programs in HSV-1 infected human cells.
\end{abstract}

Keywords: RNA-seq, Long noncoding RNA, HSV-1, In cis, In trans

\section{Background}

Approximately $70 \%$ of the human genome is transcribed, but less than $2 \%$ of which encodes proteins. The remainder, collectively referred to as noncoding RNAs, are one of the most intensely investigated subjects in almost all areas of biomedical science. Based on the size of their transcripts, noncoding RNAs (ncRNAs) are classified into

\footnotetext{
* Correspondence: zhoujm@mail.kiz.ac.cn

${ }^{1}$ Key Laboratory of Animal Models and Human Disease Mechanisms of the Chinese Academy of Sciences \& Yunnan Province, Kunming Institute of Zoology, Kunming, Yunnan 650223, China

Full list of author information is available at the end of the article
}

small noncoding RNAs (length $<200 \mathrm{nt}$ ) and long noncoding RNAs (lncRNAs, length $>200 \mathrm{nt}$ ). With their number approaching 10 thousand in the human genome [1, 2], lncRNAs have been shown to have diverse activities, ranging from recruiting chromatin remodeling complexes to transcriptional regulation and post transcriptional processing of RNAs [3]. LncRNAs could cooperate with DNA, other RNAs or proteins [4] to function in cell differentiation, development to diseases [5]. One of the best understood IncRNAs functioning in development, HOTAIR, is located within the HoxC gene cluster on chromosome 12, and represses the expression of genes in the HoxD gene cluster on chromosome 2 [6]. The most 
heavily investigated imprinted loci, IGF2/H19 encode a lncRNA H19, and its depletion caused precocious muscle differentiation [7]. lnc-DC, on the other hand, is a lncRNA implicated in immune system development, its knockdown impaired Dendritic Cell (DC) differentiation and reduced capacity of DCs to stimulate T cell activation [8]. LncRNA is also implicated in tumorigenesis, for example, a lncRNA ceruloplasmin (NRCP) was highly up-regulated in ovarian tumors, which significantly increased cancer cell growth by altering glycolysis compared with normal cells [9]. MALAT1 (metastasis associated lung adenocarcinoma transcript 1), one of the most abundant lncRNAs, could regulate alternative splicing by modulating the phosphorylation of the serine/arginine splicing factors [10]. LncRNA is also implicated in pathogen-host interaction [11], for example, a cellular lncRNA, negative regulator of antiviral response (NRAV), promoted influenza A virus (IAV) replication and virulence.

An increasing amount of evidence suggests that lncRNAs act either locally to regulate nearby genes in cis or remotely, i.e. over one mega bases away or on a different chromosome, in trans to control the transcription of target genes $[2,4,12,13]$. LncRNAs could directly silence or activate gene expression, or by indirectly regulate chromatin states of their target genes [14]. For instance, Evf, a cis-acting lncRNA, is required for the activation of Distalless homeobox (Dlx) 5 and 6 genes and generation of GABAergic interneurons in vivo [15]. HOTAIR, on the other hand, binds to PRC2 and LSD1 complexes and couples H3K27 methylation and H3K4 demethylation activity to hundreds of sites genome-wide [16].

Herpes Simplex Virus type I (HSV-1) is a double strand DNA virus, with a $152 \mathrm{~kb}$ genome, encoding about 80 genes, which also include several small RNAs and a lncRNA, latency associated transcript, or LAT $[17,18]$. HSV-1 reactivation can cause diseases from the mild cold sores to the crippling encephalitis [19]. During productive HSV-1 infection, which is responsible for these diseases, host cells activate native antiviral immunity [20,21], apoptosis $[22,23]$, DNA damage response and other stress responses [24-27] to limit HSV-1 infection and growth [27]. However, many viral genes are designed to modify these responses. For example, ICP4, ICP22 and ICP27 are negative regulators of the host apoptotic response, ICP34.5 inhibits the type I interferon response by inactivating protein kinase R (PKR) [28-30], while ICP8 inhibits the host DNA damage response by inactivating the ataxiatelangiectasia and Rad3 related (ATR) kinase [31-34].

Although the molecular details of the HSV-1 lytic infection process is well understood, many important questions on virus-host interactions and host responses, especially at the transcriptomic level still remain unanswered. Here we analyzed RNA-seq data [35] of HSV-1 infected human HFF cells for differentially expressed lncRNAs, and found
208 annotated and 206 novel lncRNAs in HSV-1 infected cells. Using method described by Derrien et al. [2], we found protein-coding genes (PCGs) that are either negatively or positively correlated with lncRNAs expression. These correlated lncRNAs exist both in cis and in trans relative to their target PCGs, and are mostly involved in chromatin assembly and metabolic process GO terms. Pathway analysis showed that PCGs correlated with lncRNAs in trans were enriched in B cell receptor signaling pathway, Toll-like receptor signaling pathway and RIG-I-like receptor signaling pathway. Pathway analysis of PCGs associated with lncRNAs in cis included axon guidance, focal adhesion, adherens junction and Neurotrophin signaling pathways. Interestingly, genes in two neurodegenerative diseases, Huntington's disease (HD) and Parkinson's disease (PD) are potentially regulated by lncRNAs, such as PPP3CB-AS1, SNHG8 and DARS-AS1. This analyses suggested important roles of lncRNAs in HSV-1 infection, and their regulation of cellular gene transcription.

\section{Methods \\ Prediction of IncRNAs}

We used FastQC software to filter low quality reads with default argument.

Clean RNA-seq data were aligned to Human reference genome (Homo_sapiens. GRCh38) with Tophat2. Cufflinks and Cuffcompare were used to assemble and compare transcripts with reference. Then we used to custome script to extract the length of transcript $\geq 200$ nucleotides, the number of exon $\geq 2$ and belonged to class code "i", "j", "o", "u" and " $x$ ". We used CNCI software to predict the coding capacity of candidate lncRNAs. Based on Sun et al. [36], we obtained high quality assemblies and used it as final reference annotation file. We counted differentially expressed genes with Cuffdiff2, and used the following criterion to select differentially expressed lncRNA genes: FDR $\leq 0.05$ and foldchange $\geq 2$ (Additional file 1: Figure S1).

\section{Targets of IncRNAs}

For gene regulation by lncRNA in cis, we extracted PCGs located within a genomic window of $1 \mathrm{Mb}$ as targets of lncRNAs. For in trans regulation of PCGs by lncRNA, we extracted PCGs far away with lncRNA about $1 \mathrm{Mb}$ or located in different chromosomes. Then we calculated the correlation coefficient (r, Pearson, $p$ value $\leq 0.05$ ) between the targets and lncRNAs and selected $|r| \geq 0.8$ and $|r| \geq 0.9$ for cis and trans, respectively (Additional file 1: Figure S1).

\section{Gene ontology and pathway analysis}

We uploaded the targets of lncRNAs into The Database for Annotation, Visualization and Integrated Discovery (DAVID) v6.7 to do Gene Ontology analyses (biological processes) and Pathway analyses (KEGG pathways). DAVID calculated a $p$ value for gene enrichment with a modified 
Fisher's exact test, and a Benjamin-Hochberg multiple test correction. We selected significant GO terms and pathways with $p$ value $\leq 0.05$.

\section{Co-expressed modules analysis}

We used STEM software to analyze co-expressed modules of PCGs and lncRNAs, and we chose significant modules with $p$ value $\leq 0.05$.

\section{Statistic analysis}

We used $R$ relative packages, such as pheatmap (pheatmap: Pretty Heatmaps, Raivo Kolde, 2015) and VennDiagram (VennDiagram: Generate High-Resolution Venn and Euler Plots, Hanbo Chen, 2015), and functions, such as cor.test to analyze data and draw figures.

\section{Results}

LncRNAs expressed in HSV-1 infected HFF cells

To determine how IncRNA expression is affected by HSV-1 infection, we analyzed RNA-seq datasets from NCBI GEO and filtered the low quality reads using FASTX-Toolkit (http://hannonlab.cshl.edu/fastx_toolkit/ ) software, followed by aligning RNA-seq data onto human reference genome with Tophat2 [37], and subsequently using Cufflinks [38] and Cuffcompare [38] to assemble and compare transcripts. In addition, we extracted the fasta sequences of candidate novel lncRNAs, and used CNCI [39] software to distinguish noncoding RNAs from coding RNAs. Finally, compared to Ensembl database, we obtained 14,654 annotated lncRNAs and 3,050 novel lncRNAs consisted of 5,909 transcripts (Additional file 1: Figure S1).

Previous studies in mammals showed that lncRNAs are shorter in length, have fewer exons and expressed at much lower levels than PCGs [1, 36, 40, 41]. To determine whether the novel lncRNAs we detected have the same features, we calculated exon numbers in detected novel lncRNAs and PCGs, and found that the exon numbers of these novel lncRNAs are much smaller than that of PCGs ( $p$ value $<0.05$, Welch Two Sample t-test) (Fig. 1a), and the exon length of novel lncRNAs is much shorter than that of PCGs ( $p$ value $<0.05$, Welch Two Sample t-test) (Fig. 1b). We next calculated the expression level of PCGs and novel lncRNAs, and found that the expression level of PCGs is much higher than that of novel lncRNAs in all samples with the exception of 6hpi and 8hpi samples $(p$ value $<0.05$, Welch Two Sample ttest, Fig. 1c). The expression levels of PCGs from the 6hpi and 8hpi samples were slightly higher than that of novel lncRNAs, which is likely due to the viral protein VHS, which is known to degrade host mRNAs [42-46] ( $p$ value $<0.05$, Welch Two Sample t-test). Thus, these novel, de novo assembled lncRNAs are highly credible.
We then used the high quality GTF file as reference annotation file for further analysis.

Differentially expressed IncRNAs due to HSV-1 infection Cuffdiff2 [47] software was used to calculate differentially expressed (DE) genes from 2hpi to 8 hpi samples compared to mock infected samples.

Overall, the number of DE lncRNAs increased with time after virus infection. For example, there was no DE lncRNAs at 2 hours after HSV-1 infection, but there were 15 annotated and 17 novel DE lncRNAs expressed at 4hpi sample (Fig. 2a and b). At 6hpi sample, 74 annotated and 75 novel DE lncRNAs were found, respectively (Fig. 2a and b), while in the 8hpi sample, many more DE lncRNAs, including 193 annotated and 197 novel lncRNAs were observed (Fig. 2a and b). We pooled all DE lncRNAs from 4hpi to 8hpi samples, and obtained 414 DE lncRNAs, including 208 annotated and 206 novel lncRNAs (Fig. 2a and b).

Based on methods by Derrien et al. [2], we also classified the above DE lncRNAs into five types: antisense RNA, long intronic non coding RNA (lincRNA), processed transcript, sense intronic transcript, and sense overlapping transcript. We found that the number of lincRNAs was most abundant among all lncRNAs. Previous study showed the expression of lncRNAs is very cell type and tissues specific [2], we therefore determined the temporal specificity of lncRNAs during the course of infection. Indeed, these IncRNA expression patterns are highly dynamic, for example, there were only 27 overlapped DE lncRNAs, including 11 annotated and 16 novel lncRNAs, when compared the 4hpi with 8hpi samples (Fig. 2a and d). This dynamic nature is consistent with their regulatory roles.

\section{Prediction of cis target genes of virus induced DE IncRNAs}

Based on previous studies describing in cis and in trans regulation modes to predict target PCGs and functions of lncRNAs at transcriptomic level $[2,48]$, we analyzed in cis correlation coefficient (r) of expression of lncRNAs and PCGs. Between PCGs and lncRNAs, we obtained 928 and 1,188 pairs of positive and negative regulatory modes, respectively (Fig. 3a and b).

Using DAVID [49] software, Gene Ontology (GO) analysis of DE PCGs with positive cis correlation coefficients with lncRNAs revealed genes enrichment for metabolic processes and neuronal differentiation GO terms (Fig. 3c). In contrast, PCGs negatively correlated with lncRNAs were enriched in assembly of marco molecular complexes, such as nucleosome and chromatin assembly. For example, two PCGs, HIST1H2AB and HIST1H2AC, which were both significantly down-regulated about 2.5 fold after HSV-1 infection, were negatively associated with one annotated 

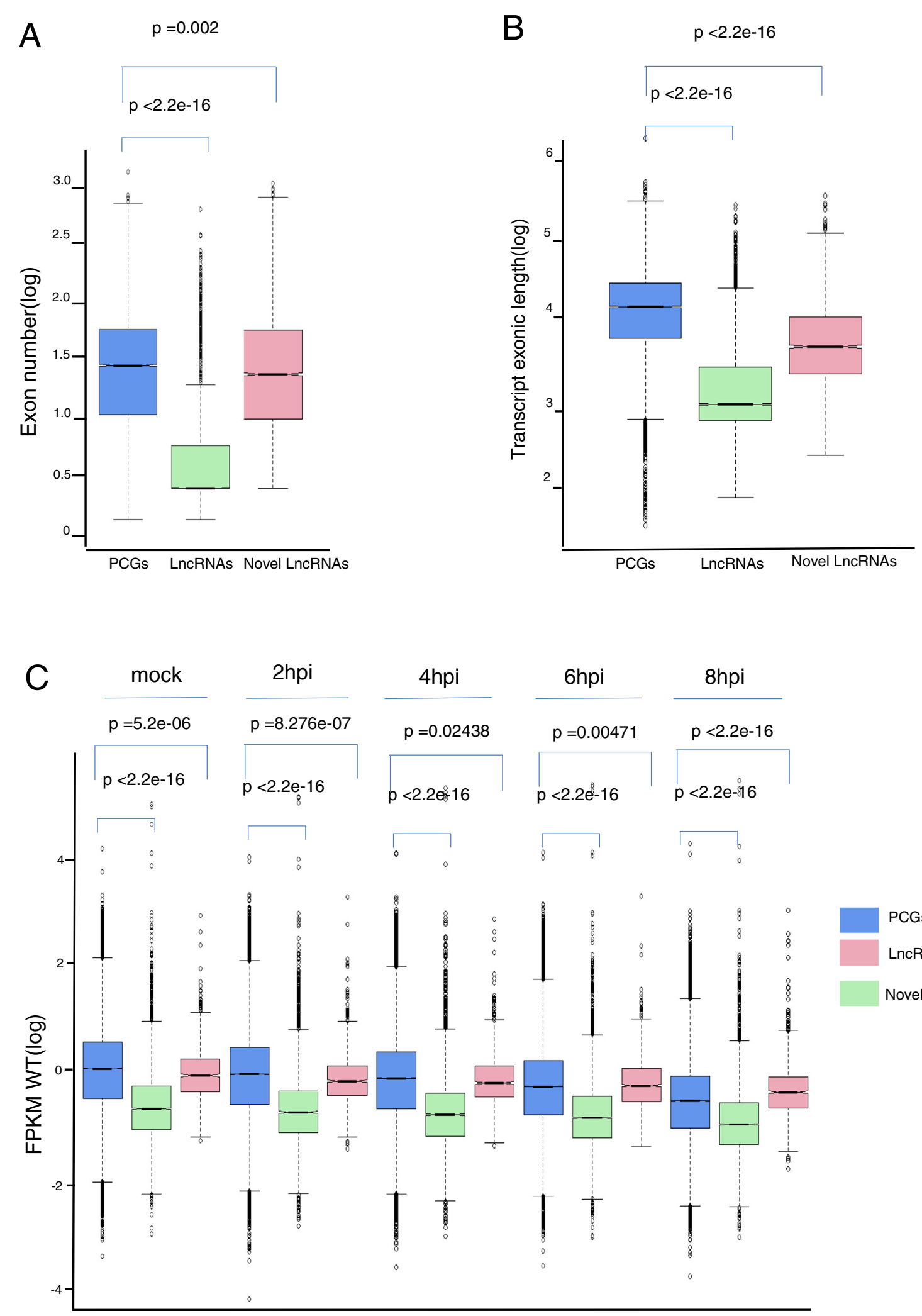

Fig. 1 (See legend on next page.) 
(See figure on previous page.)

Fig. 1 Comparisons of transcript length, exon number and expression levels of HSV-1 infected transcriptome. a Comparison of exon number. The novel IncRNAs represent smaller the number of exon than PCGs on average. $\mathbf{b}$ Comparison of transcript length. The novel IncRNAs show shorter length on average than PCGs. c The coding transcripts represent slightly higher expression level than the novel IncRNAs, but significantly higher expression than the annotated IncRNAs. Wilcox.test, $p$ value $<0.05$

lncRNA, ZNRD1-AS1 (significantly up-regulated about 4 fold by the infection) (Fig. 3d).

Pathway (KEGG) enrichment analysis revealed 9 pathways for DE PCGs positively affected by lncRNAs in cis, and 10 pathways for DE PCGs negatively affected by lncRNAs in cis (Fig. 3e and f), which include axon guidance, focal adhesion, adherens junction and Neurotrophin signaling pathways. These pathways are involved in differentiation and survival of neural cells, and higher neuronal function, such as learning and memory [50, 51]. As HSV-1 is a neurotropic virus, the regulation of these genes in cis by HSV-1 induced lncRNAs is of biological significance.
One of the immediate early viral protein encoded by HSV1 , ICP0 is known to activate neuronal genes [25, 52-54], and may contribute to the DE lncRNAs and correlated PCGs. Interestingly, we also found PCGs with negative correlation coefficients were enriched in Systemic lupus erythematous, which is reported to be linked to HSV-1 infection [55].

To confirm the co-expression signature of PCGs and lncRNAs by correlation analyses, we used STEM [56] software to analyze co-expressed modules from the above-mentioned expression of PCGs and lncRNAs. In both positive and negative regulatory modules, we obtain
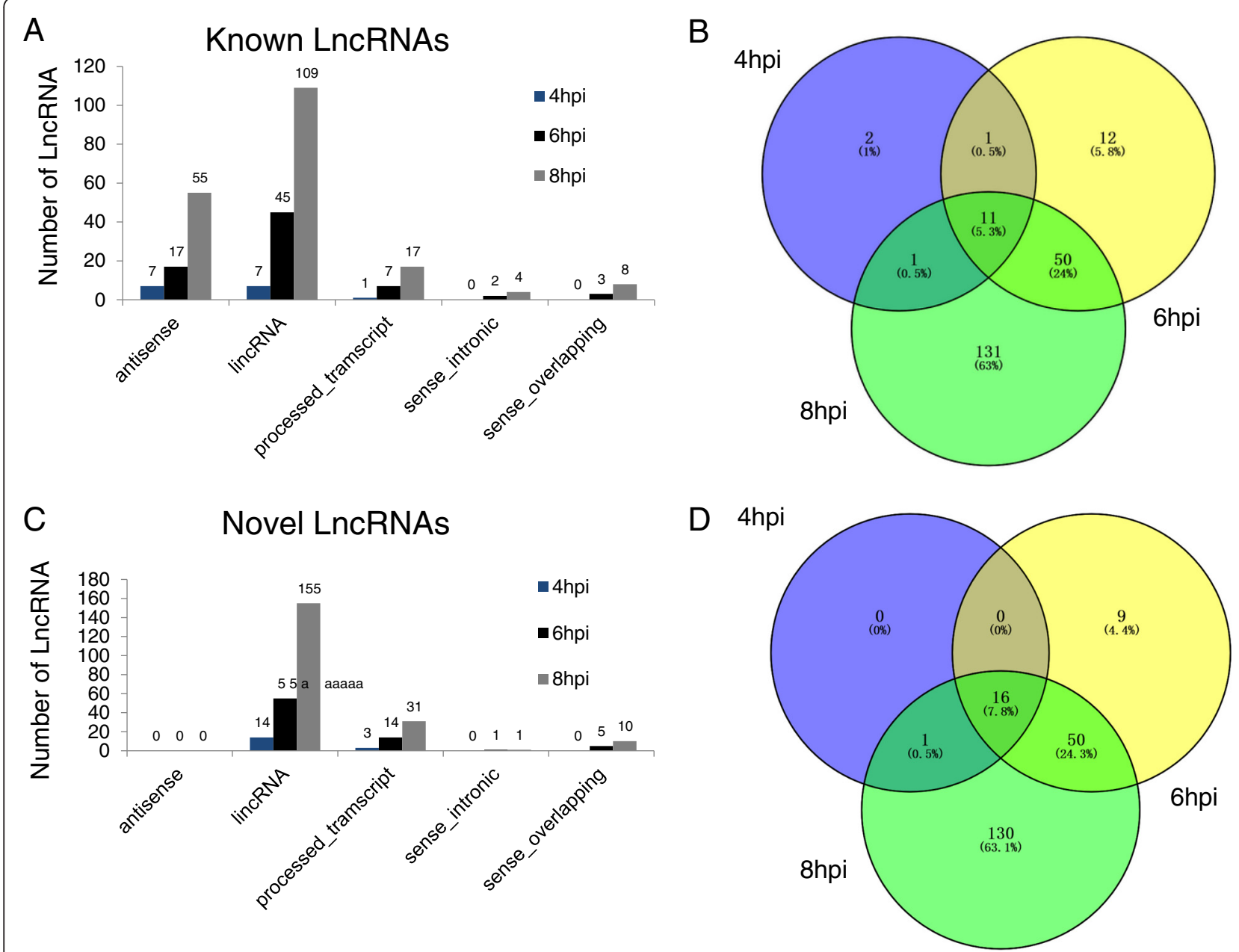

Fig. 2 The number of differentially expressed IncRNAs at different time points after HSV-1 infection. a There are 15, 74 and 193 annotated differentially expressed IncRNAs in 4hpi, 6hpi and 8hpi samples, respectively. b There are 11 common annotated IncRNAs among 4hpi, 6hpi and 8hpi samples. c There are 17,75 and 197 differentially expressed novel IncRNAs in 4hpi, 6hpi and 8hpi samples, respectively. d 16 common novel IncRNAs are found among 4hpi, 6hpi and 8hpi samples 


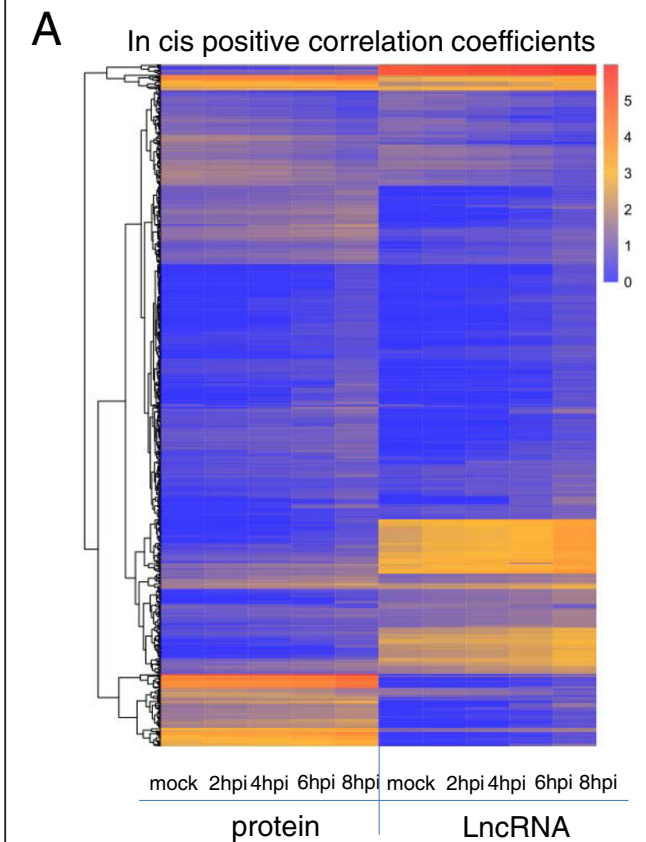

B

In cis negative correlation coefficients

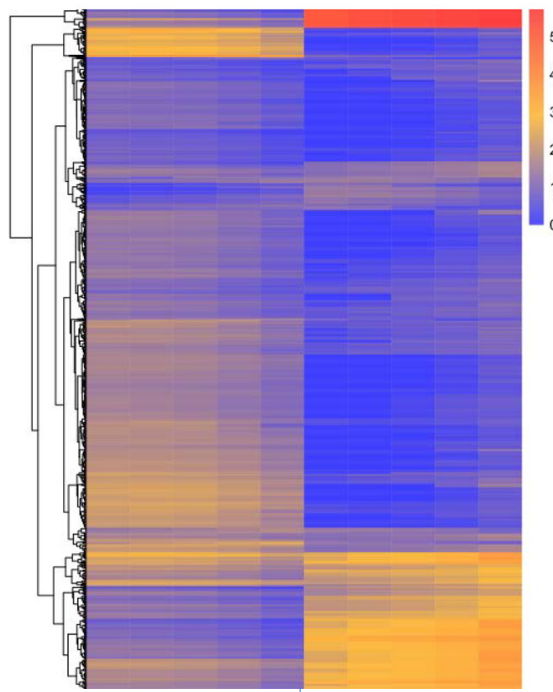

mock 2hpi 4hpi 6hpi 8hpi mock 2hpi 4hpi 6hpi 8hpi

$$
\text { protein } \quad \text { LncRNA }
$$

C

D

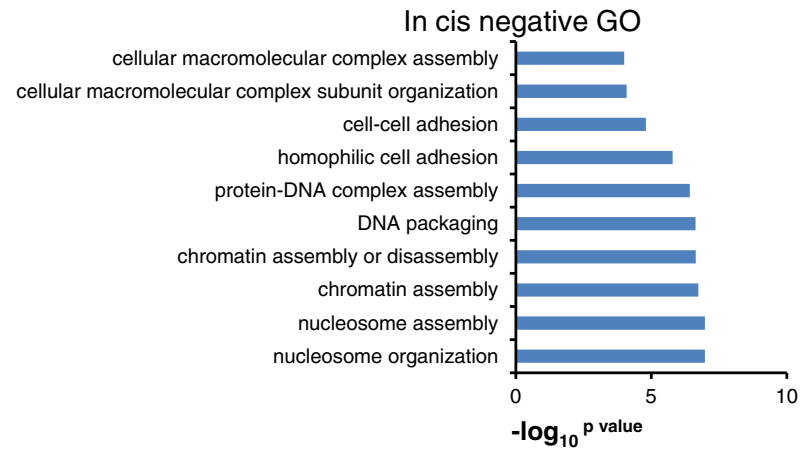

$E$

In cis positive pathway

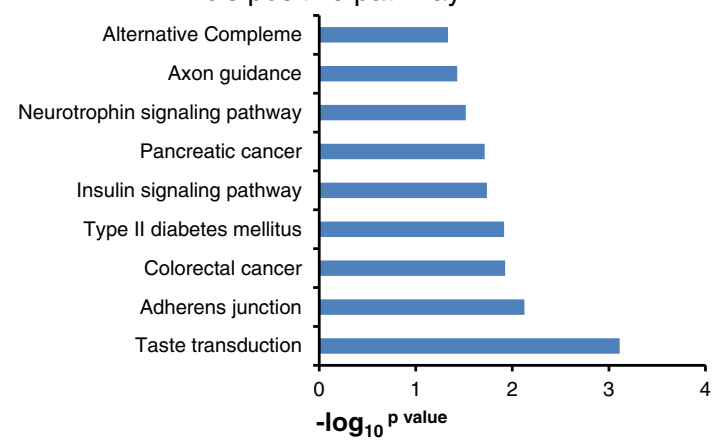

$\mathrm{F}$

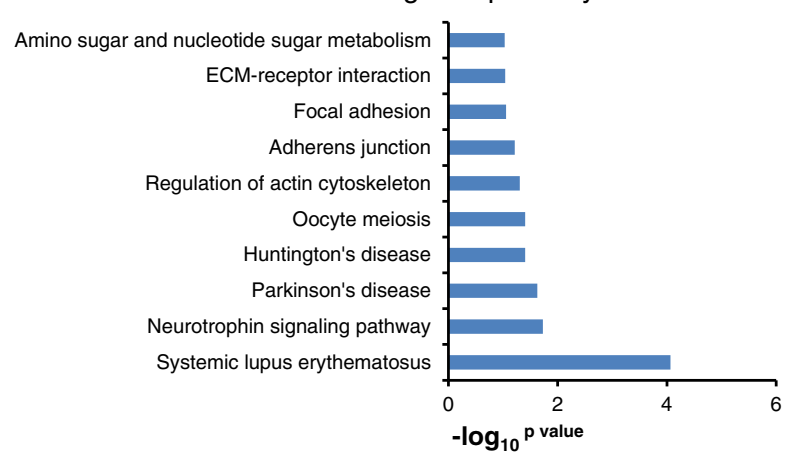

Fig. 3 The predicted function of IncRNAs regulating PCGs in cis. a 714 PCGs and 83 IncRNAs composed 918 pairs of positive in cis, the color-axis of heatmap is the $\log _{10}^{\mathrm{FPKM}+1}$. The correlation coefficients $(r) \geq 0.8, p$ value $\leq 0.05$. b 933 and 84 IncRNAs composed 1188 negative regulatory modes in cis. The correlation coefficients $(r) \leq-0.8, p$ value $\leq 0.05$. $\mathbf{c}$, e. $\mathrm{GO}$ analysis and pathway analysis of coding genes associated with positive correlation coefficients, $p$ value $\leq 0.05 ;(\mathbf{d}, \mathbf{f})$. GO analysis and pathway analysis of PCGs associated with negative correlation coefficients, $p$ value $\leq 0.05$. The $x$-axis of $c / d / e / f$ graphs is the $-\log _{10} p$ value 
5 and 4 specific profiles showed significant enrichment for PCGs and lncRNAs ( $p$ value $<0.05$ ), respectively (Additional file 1: Figure S2A and D). GO and Pathway enrichment analysis for these PCGs from significant coexpressed modes (Additional file 1: Figure S2A and C, E and F) showed that the top10 GO terms and pathways were also enriched in chromatin assembly, metabolic processes and neuronal differentiation, and Neurotrophin signaling and adherens junction, respectively. Thus, these results are consistent with the results obtained using correlation analysis.

\section{Prediction of trans target genes of HSV-1 induced DE IncRNAs}

We then analyzed in trans correlations of expression (defined as pairs consisting of lncRNAs and coding genes separated by a distance of $>1$ mega base, or located on different chromosomes), and found 2,072 and 1,803 pairs of positive and negative regulatory modes, respectively (Fig. $4 \mathrm{a}$ and b).

$\mathrm{GO}$ analysis of genes with positive correlation coefficients enriched these genes in transcription, metabolic processes, and development on the top 10 terms (Fig. 4c). For example, one PCG, KDM5B, a lysine-specific histone demethylase from the jumonji/ARID domain-containing family with a role in the transcriptional repression [57], was up-regulated after HSV-1 infection and showed highly positive correlation $(r>0.9, p$ value $<0.01)$ with one annotated IncRNA, KCNQ1OT1. KCNQ1OT1 interacts with chromatin and regulates transcription of multiple target genes through epigenetic modifications [58]. On the other hand, the coding gene, PRKAA1, a member of Ser/Thr protein kinase family [59], positively associated with one novel lncRNA (location of the novel lncRNA in genome: Chr22: 38705722-38794198), which was up-regulated after HSV-1 infection. Again from the imprinted IGF2/H19 loci, IGF2 [60] was up-regulated by HSV-1 infection, and showed highly positive correlation with one antisense lncRNA, BZRAP1-AS1. These positive correlations strongly suggest regulatory connections between lncRNAs and correlated PCGs in chromatin and transcriptional regulation.

Interestingly, we also found genes positively affected by lncRNAs through in trans were enriched in B cell receptor signaling pathway, which resulted in the expression of immediate early genes that further activated the expression of other genes involved in B cell proliferation, differentiation and Ig production as well as other processes [61]. For example, B-cell linker, BLNK, encodes a cytoplasmic linker or adaptor protein that plays a critical role in B cell development [62], was induced after HSV-1 infected HFF cells for $6 \mathrm{~h}$. It exhibited highly positive correlation $(r>$ $0.99, p$ value $<0.001$ ) with two novel lncRNAs (located at Chr12:220425-262873 and Chr9:99297947-99319599).
Another important enriched pathway is the Toll-like receptor signaling pathway [63]. For example, IRF3, which was significantly down-regulated by HSV-1 infection, associated with one novel lncRNA (located at Chr7: 34,928,699$35,038,271)$, while IRF5 and IRF7, significantly up-regulated after HSV-1 infected for $8 \mathrm{~h}$, were positively associated with two annotated lncRNAs, ZNRD1-AS1 and MAMDC2AS1, respectively. A third immunity related pathway is the RIG-I-like receptor signaling pathway, which is responsible for detecting viral pathogens and generating innate immune responses [64, 65] (Fig. 4e). Besides immune genes, we also found lncRNAs functioning in the DNA damage response, for example, protein coding gene ATG5, which was significantly down-regulated after HSV-1 infection, showed positive association with one lncRNA, noncoding RNA activated by DNA damage (NORAD), which is also down-regulated after HSV-1 infection [11]. As HSV-1 infection is known to activate the cellular DNA damage response, the down regulation of NORAD could be due to the active inhibition of one viral gene, ICP8, which inhibits the host DNA damage response by inactivating the ATR kinase [31-34].

In contrast, genes with negative correlation coefficients with lncRNAs were enriched in apoptosis and cell proliferation by GO analysis (Fig. 4d), suggesting that genes in these two processes are likely subject to inhibition by virus induced lncRNAs. For example, CASP7, a member of caspases functioning in apoptosis [66], was down-regulated by HSV-1 infection, and showed negative correlation with three lncRNAs, PPP3CB-AS1, SNHG8 and DARS-AS1. TGFBR2, a member of the Ser/ Thr protein kinase family and the TGFB receptor subfamily [67], was down-regulated after HSV-1 infection and showed highly negative correlation $(r<-0.9, p$ value $<0.01)$ with KCNQ1OT1, which can interact with chromatin and regulate transcription of multiple target genes through epigenetic modifications [50].

Notably, two neurodegenerative diseases, Huntington's disease (HD) and Parkinson's disease (PD) pathway genes are negatively correlated with lncRNAs both in cis and in trans (Fig. 3e and Fig. 4f). HD, a neurodegenerative genetic disorder, can affect muscle coordination and lead to mental decline and behavioral symptoms [68]. Previous studies found that REST (RE1-Silencing Transcription Factor) is involved in HD and is considered a hub in the co-ordinate regulation of the transcriptome and epigenome in HD [69, 70]. Normally, REST represses neuronal genes in non-neuronal tissues [71], but in HSV-1 infected HFF cells, it was significantly down-regulated, leading to up-regulation of many neuronal genes, which is believed to be caused by HSV-1 protein ICP0 [25, 52-54]. $\mathrm{PD}$ is another important neurodegenerative disorder [72]. Here UCHL1 (Ubiquitin Carboxyl-Terminal Esterase L1), a susceptibility gene for PD and a potential target for 
A In trans positive correlation coefficients

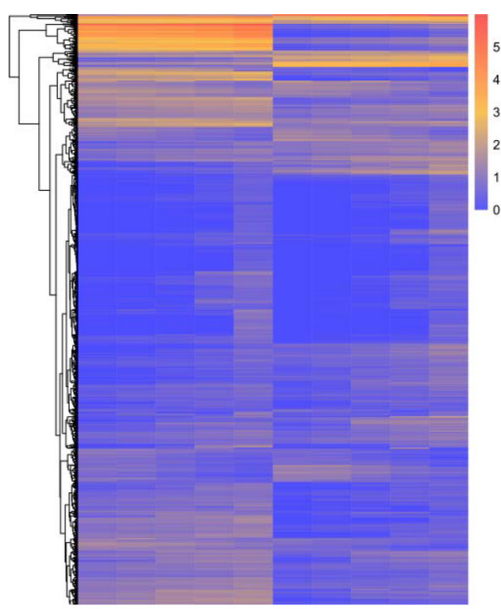

mock 2hpi 4hpi 6hpi 8hpi mock 2hpi 4hpi 6hpi 8hpi protein $\quad$ LncRNA

B

In trans negative correlation coefficients

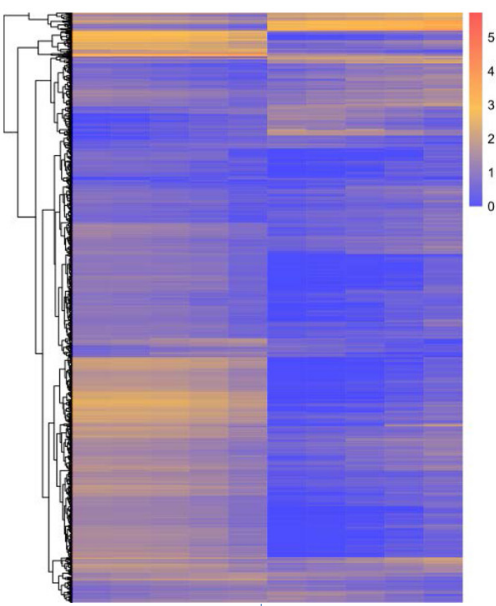

mock 2hpi 4hpi 6hpi 8hpi mock 2hpi 4hpi 6hpi 8hpi protein LncRNA
C

In trans positive GO

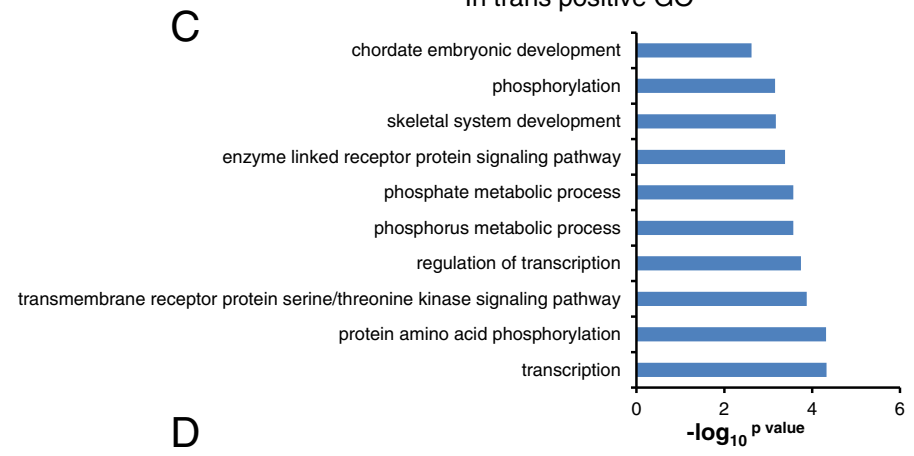

In trans negative GO

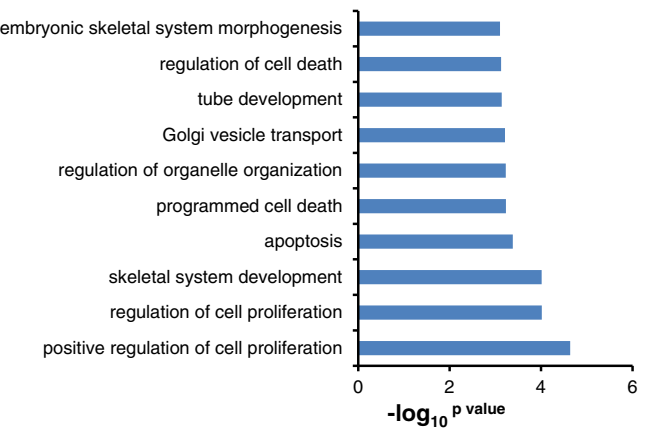

In trans positive pathway

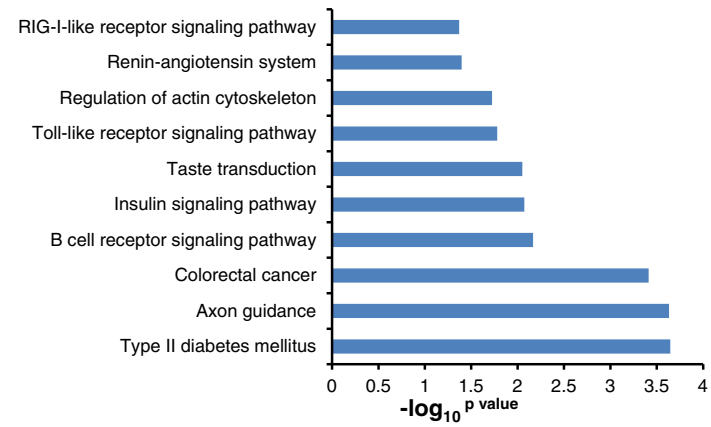

In trans negative pathway

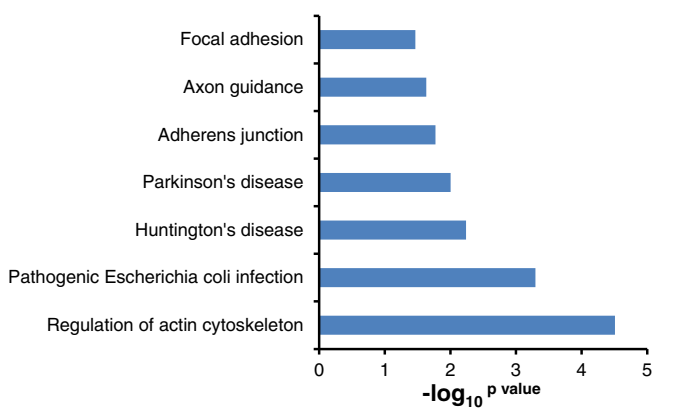

Fig. 4 The predicted function of IncRNAs regulating PCGs in trans. a 1029 PCGs and 387 IncRNAs composed 2072 pairs of positive in trans, the color-axis of heatmap is the $\log _{10}^{\text {FPM }+1}$. The correlation coefficients $(r) \geq 0.9, p$ value $\leq 0.05$. b 1047 and 369 IncRNAs composed 1803 pairs of negative regulatory modes in trans. The correlation coefficients $(r) \leq-0.9, p$ value $\leq 0.05$. $\mathbf{c}-\mathbf{d} \mathrm{GO}$ analysis and pathway analysis of coding genes associated with negative correlation coefficients, $p$ value $\leq 0.05$; (e-f). $\mathrm{GO}$ analysis and pathway analysis of PCGs associated with positive correlation coefficients, $p$ value $\leq$ 0.05. The $x$-axis of $c / d / e / f$ graphs is the $-\log _{10} p$ value 
disease-modifying therapies [73], was significantly downregulated by HSV-1 infection, and a novel, HSV-1 induced lncRNA (located at 4:68282460-68285959), negatively correlated with REST and UCHL1, thus could potentially inhibit the expression of REST and UCHL1. Therefore, HSV-1 induced lncRNAs could promote neural specific programs.

We also analyzed co-expressed modules of PCGs and lncRNA to check whether the correlation analysis is right, we found 8 and 6 significant co-expressed modules for positive and negative regulation $(p$ value $<0.05)$, respectively (Additional file 1: Figure S3A, D). Compared to above mentioned GO terms, this method found that PCGs, co-expressed with IncRNAs, were also enriched in the similar GO terms, such as metabolic processes and development. In addition, pathway enrichment analysis revealed that many PCGs were also enriched in the pathways detected by correlation analysis. For instance, we found that some PCGs, such as BLNK, REST and UCHL1, were enriched in B cell receptor signaling pathway, $\mathrm{HD}$ and $\mathrm{PD}$, respectively (Additional file 1: Figure S3B and C, E and F).

\section{Discussion and conclusions}

HSV-1 is an important and ubiquitous human pathogen. Its lytic infection in cultured cells has been used as a paradigm to investigate the basic mechanism of transcription, molecular virology and virus-host interactions. HSV-1 infection is known to profoundly alter the host transcriptome, from differential gene expression, to RNA splicing and RNA Pol II read through [29, 35, 74]. The human genome encodes around ten thousand lncRNAs $[16,17]$, which are involved in many different biological processes $[3,5]$. However, how lncRNA expression is affected by HSV-1 infection and whether lncRNAs play any roles in the transcriptomic response to viral infection are not understood. Here we analyzed the HSV-1 infected transcriptome to reveal how many lncRNAs are expressed in HFF cells and how they are affected by the infection, and found 14,654 annotated lncRNAs, and 3,050 novel lncRNAs when compared to the Ensemble database. Among the 3,050 newly discovered lncRNAs, 789 were induced by HSV-1 infection. Then based on criterion (FDR $\leq 0.05$ and fold-change $\geq 2$ ) of differential expression analysis, we obtained 208 annotated DE lncRNAs, including 166 up-regulated and 42 downregulated lncRNAs, and 206 novel DE lncRNAs, including 171 up-regulated and 35 down-regulated lncRNAs, after HSV-1 infection. Similar method of predicting novel lncRNAs was used by Sun et al. [36], in order to avoid genome contamination in RNA-seq samples, here we set more strict cutoff that the number of exon of candidate novel lncRNAs is more than 1. As a result, our prediction may have excluded some positive genes.
Next, we predicted the potential targets of the $\mathrm{DE}$ lncRNAs by correlation of expression levels of PCGs and lncRNAs. An important modus operandi of lncRNAs is to act in cis to control the expression of PCGs that are positioned in the vicinity of their transcription sites [5]. By correlation prediction of expression levels in cis mode, we found many PCGs negatively associated with lncRNAs were enriched in large marco molecular complex assembly, including protein DNA complex assembly, nucleosome assembly and chromatin assembly, suggesting that these IncRNAs may negatively regulate these PCGs. In contrast, GO analysis showed that PCGs positively correlated with lncRNAs were enriched in metabolic process, differentiation and phosphorylation. These patterns of correlations suggest that these lncRNAs might regulate the correlated PCGs in these biological processes.

HSV-1 is well known to modulate host responses from native immunity $[29,75]$ to apoptosis $[17,18]$ to benefit viral transcription and replication. Pathway analysis showed that some PCGs positively related with lncRNAs in trans were enriched in B cell receptor signaling pathway, Tolllike receptor signaling pathway and RIG-I-like receptor signaling pathway. For instance, IRF5 and IRF7, positively correlated with ZNRD1-AS1 and MAMDC2-AS1, may be up-regulated by two lncRNAs, respectively. This analysis suggests that lncRNA might play important roles in regulating immune response. In the list of genes that are predicted to be in trans targets of IncRNAs, we found that PCGs negatively associated with lncRNAs were enriched in apoptosis. For example, PIM2, reported to promote cell survival and inhibit apoptosis [76], was up-regulated by HSV-1 in HFF cells and was negatively correlated with lncRNA DNM3OS. Meanwhile, CASP7, a member of the apoptotic pathway [61], was down-regulated by HSV-1 infection and showed negative correlation with PPP3CB-AS1, SNHG8 and DARS-AS1. Thus, this analysis brings forward a hypothesis that HSV-1 might modulate the host apoptotic pathway by targeting IncRNAs, including DNM3OS, PPP3CB-AS1, SNHG8 and DARS-AS1.

The DNA damage response is another important cellular response to HSV-1 infection. Here ATG5, which functions as a potent molecular decoy for PUMILIO proteins repress a program of genes necessary to maintain genomic stability [11], was significantly down regulated after HSV-1 infected in HFF cells. This expression pattern showed highly positive association with NORAD [10]. HSV-1 infection induces DNA replication stress and activates the DNA damage response [24-26]. At the same time, viral protein ICP8 inhibits the ATR kinase activity to modulate the cellular DNA damage response [31-34]. Thus it would be interesting to investigate whether HSV-1 actively inhibits the expression of NORAD, for example by ICP8 to modulate host response. 
In summary, we predicted potential target genes of HSV-1 induced, differentially expressed lncRNAs in HFF cells, and revealed a large number of target genes that may participate in cellular pathway functions in response to viral infection. Our analysis suggests that one lncRNA might regulate many PCGs, while individual PCGs could also be regulated by multiple IncRNAs. The two methods we used, correlation analysis and co-expression module analysis by and large produced similar results, thus these potential lncRNAs target genes offer important clues to further study mechanism of viral-host interaction and the regulatory functions of lncRNAs.

\section{Additional file}

Additional file 1: Figure S1. Computational prediction of IncRNAs in HSV-1 infected HFF cells. The computational strategy of predicting of function of IncRNAs in HSV-1 infected HFF cells. Figure S2 The predicted function of IncRNAs based on co-expressed modules in cis. a. there were 5 significant co-expressed modules in positive regulatory model, $p$ value $\leq$ 0.05. b-c. GO analysis and pathway analysis of PCGss co-expressed with IncRNAs, $p$ value $\leq 0.05$; $d$. there were 4 significant co-expressed modules in negative regulatory model, $p$ value $\leq 0.05$. e-f. GO analysis and pathway analysis of PCGs co-expressed with IncRNAs, $p$ value $\leq 0.05$. Figure $S 3$ The predicted function of IncRNAs based on co-expressed modules in trans. a. there were 8 significant co-expressed modules in positive regulatory model, $p$ value $\leq 0.05$. b-c. GO analysis and pathway analysis of PCGss co-expressed with IncRNAs, $p$ value $\leq 0.05$; $d$. there were 6 significant co-expressed modules in negative regulatory model, $p$ value $\leq 0.05$. e-f. GO analysis and pathway analysis of PCGs co-expressed with IncRNAs, $p$ value $\leq 0.05$. (DOCX $653 \mathrm{~kb}$ )

\section{Abbreviations}

$\mathrm{DE}$, differentially expressed; $\mathrm{GO}$, gene ontology; HD, huntington's disease; HFF, human foreskin fibroblast; HSV-1, herpes simplex virus type i; IncRNA, long noncoding RNA; PCGs, protein-coding genes; PD, parkinson's disease

\section{Acknowledgements}

Acquisition of data, analysis, interpretation of data and manuscript writing: Benxia Hu; Data analysis: Yongxia Huo; Manuscript writing: Guijun Chen, Liping Yang and Dongdong Wu; Manuscript writing and revised it critically for important intellectual content: Jumin Zhou. This work was in part supported by grants from National Science Foundation of China provided funding to Jumin Zhou under grant number NSFC81471966. Kunming Institute of Zoology, Chinese Academy of Sciences provided funding to Jumin Zhou under grant number Y102421081. Yunnan Provincial Government provided funding to Jumin Zhou under grant numbers 2011 HA005 and 2013FA051. Chinese Academy of Sciences provided funding to Jumin Zhou under grant number KSCXZ-EW-BR-6.

\section{Funding information}

National Science Foundation of China provided funding to Jumin Zhou under grant number NSFC81471966. Kunming Institute of Zoology, Chinese Academy of Sciences provided funding to Jumin Zhou under grant number Y102421081. Yunnan Provincial Government provided funding to Jumin Zhou under grant numbers 2011HA005 and 2013FA051. Chinese Academy of Sciences provided funding to Jumin Zhou under grant number KSCXZ-EW-BR-6.

\section{Availability of data and materials}

RNA-seq datasets of HSV-1 infected HFF cells with multiplicity of infection (MOI) of 10 were downloaded from NCBI Gene Expression Omnibus (GEO) database with accession number GSE59717, which included 2 replicates for each experiment (mock, 2, 4, 6 and 8 h post infection (hpi)).

\section{Authors' contributions}

$\mathrm{BH}$ carried out data analysis and manuscript writing. YH carried out data analysis. GC participated in manuscript writing. LP participated in manuscript writing. DW participated in manuscript writing. JZ participated in experiment design and manuscript writing. All authors read and approved the final manuscript.

\section{Authors' information}

Jumin Zhou is a principal investigator of Laboratory of Epigenetics and Gene Regulation.

\section{Competing interests}

The authors declare that they have no competing interests.

\section{Author details}

${ }^{1}$ Key Laboratory of Animal Models and Human Disease Mechanisms of the Chinese Academy of Sciences \& Yunnan Province, Kunming Institute of Zoology, Kunming, Yunnan 650223, China. ${ }^{2}$ Kunming College of Life Science, University of Chinese Academy of Sciences, Kunming, Yunnan 650204, China. ${ }^{3}$ State Key Laboratory of Genetic Resources and Evolution, Kunming Institute of Zoology, Chinese Academy of Sciences, Kunming, Yunnan 650223, China.

Received: 3 May 2016 Accepted: 1 August 2016

Published online: 05 August 2016

\section{References}

1. Cabili MN, Trapnell C, Goff L, Koziol M, Tazon-Vega B, Regev A, Rinn JL. Integrative annotation of human large intergenic noncoding RNAs reveals global properties and specific subclasses. Genes Dev. 2011;25:1915-27.

2. Derrien T, Johnson R, Bussotti G, Tanzer A, Djebali S, Tilgner H, Guernec G, Martin D, Merkel A, Knowles DG, et al. The GENCODE v7 catalog of human long noncoding RNAs: analysis of their gene structure, evolution, and expression. Genome Res. 2012;22:1775-89.

3. Baker M. Long noncoding RNAs: the search for function. Nat Methods. 2011; 8:379-83.

4. Rinn JL, Chang HY. Genome regulation by long noncoding RNAs. Annu Rev Biochem. 2012:81:145-66.

5. Fatica A, Bozzoni I. Long non-coding RNAs: new players in cell differentiation and development. Nat Rev Genet. 2014;15:7-21.

6. Rinn JL, Kertesz M, Wang JK, Squazzo SL, Xu X, Brugmann SA, Goodnough LH, Helms JA, Farnham PJ, Segal E, Chang HY. Functional demarcation of active and silent chromatin domains in human HOX loci by noncoding RNAs. Cell. 2007;129:1311-23.

7. Kallen AN, Zhou XB, Xu J, Qiao C, Ma J, Yan L, Lu L, Liu C, Yi JS, Zhang H, et al. The imprinted H19 IncRNA antagonizes let-7 microRNAs. Mol Cell. 2013; 52:101-12.

8. Wang P, Xue Y, Han Y, Lin L, Wu C, Xu S, Jiang Z, Xu J, Liu Q, Cao X. The STAT3-binding long noncoding RNA Inc-DC controls human dendritic cell differentiation. Science. 2014:344:310-3.

9. Rupaimoole R, Lee J, Haemmerle M, Ling H, Previs RA, Pradeep S, Wu SY, Ivan C, Ferracin M, Dennison JB, et al. Long noncoding RNA ceruloplasmin promotes cancer growth by altering glycolysis. Cell Rep. 2015;13:2395-402.

10. Tripathi V, Ellis JD, Shen Z, Song DY, Pan Q, Watt AT, Freier SM, Bennett CF, Sharma A, Bubulya PA, et al. The nuclear-retained noncoding RNA MALAT1 regulates alternative splicing by modulating SR splicing factor phosphorylation. Mol Cell. 2010;39:925-38.

11. Ouyang J, Zhu X, Chen Y, Wei H, Chen Q, Chi X, Qi B, Zhang L, Zhao Y, Gao GF, et al. NRAV, a long noncoding RNA, modulates antiviral responses through suppression of interferon-stimulated gene transcription. Cell Host Microbe. 2014;16:616-26.

12. Batista PJ, Chang HY. Long noncoding RNAs: cellular address codes in development and disease. Cell. 2013;152:1298-307.

13. Guttman M, Rinn JL. Modular regulatory principles of large non-coding RNAs. Nature. 2012;482:339-46.

14. Quinodoz S, Guttman M. Long noncoding RNAs: an emerging link between gene regulation and nuclear organization. Trends Cell Biol. 2014;24:651-663.

15. Bond AM, VanGompel MJW, Sametsky EA, Clark MF, Savage JC, Disterhoft JF, Kohtz JD. Balanced gene regulation by an embryonic brain ncRNA is critical for adult hippocampal GABA circuitry. Nat Neurosci. 2009;12:1020-U1091.

16. Chu C, Qu K, Zhong FL, Artandi SE, Chang HY. Genomic maps of long noncoding RNA occupancy reveal principles of RNA-chromatin interactions. Mol Cell. 2011;44:667-78. 
17. Whitley RJ, Roizman B. Herpes simplex virus infections. Lancet. 2001;357: 1513-8.

18. Roizman B, Whitley RJ. An inquiry into the molecular basis of HSV latency and reactivation. Annu Rev Microbiol. 2013;67:355-74.

19. Padgett DA, Sheridan JF, Dorne J, Berntson GG, Candelora J, Glaser R. Social stress and the reactivation of latent herpes simplex virus type 1. Proc Natl Acad Sci. 1998;95:5.

20. Moran TP, Vickery BP. Impaired intrinsic immunity to HSV-1 in human IPSCderived TLR3-deficient CNS cells. Pediatrics. 2013;132:S51-2.

21. Griffiths SJ, Koegl M, Boutell C, Zenner HL, Crump CM, Pica F, Gonzalez O, Friedel CC, Barry G, Martin K. A systematic analysis of host factors reveals a Med23-interferon-lambda regulatory axis against herpes simplex virus type 1 replication. PLoS Pathog. 2013;9:e1003514.

22. Prasad A, Remick J, Zeichner SL. Activation of human herpesvirus replication by apoptosis. J Virol. 2013;87:10641-50.

23. Wang X, Patenode C, Roizman B. US3 protein kinase of HSV-1 cycles between the cytoplasm and nucleus and interacts with programmed cell death protein 4 (PDCD4) to block apoptosis. Proc Natl Acad Sci U S A. 2011;108:14632-6.

24. Smith S, Reuven N, Mohni KN, Schumacher AJ, Weller SK. Structure of the herpes simplex virus 1 genome: manipulation of nicks and gaps can abrogate infectivity and alter the cellular DNA damage response. J Virol. 2014;88:10146-56.

25. Lilley CE, Chaurushiya MS, Boutell C, Everett RD, Weitzman MD. The intrinsic antiviral defense to incoming HSV-1 genomes includes specific DNA repair proteins and is counteracted by the viral protein ICPO. PLoS Pathog. 2011;7: e1002084.

26. Volcy K, Fraser NW. DNA damage promotes herpes simplex virus-1 protein expression in a neuroblastoma cell line. J Neurovirol. 2013;19:57-64.

27. Negorev DG, Vladimirova OV, Ivanov A, Rauscher 3rd F, Maul GG. Differential role of Sp100 isoforms in interferon-mediated repression of herpes simplex virus type 1 immediate-early protein expression. J Virol. 2006;80:8019-29.

28. Wang $Y$, Yang $Y$, Wu SF, Pan S, Zhou CD, Ma YJ, Ru YX, Dong SX, He B, Zhang CZ, Cao YJ. p32 is a novel target for viral protein ICP34.5 of herpes simplex virus type 1 and facilitates viral nuclear egress. J Biol Chem. 2014; 289:35795-805.

29. Pasieka TJ, Baas T, Carter VS, Proll SC, Katze MG, Leib DA. Functional genomic analysis of herpes simplex virus type 1 counteraction of the host innate response. J Virol. 2006;80:7600-12.

30. Rasmussen SB, Horan KA, Holm CK, Stranks AJ, Mettenleiter TC, Simon AK, Jensen SB, Rixon FJ, He B, Paludan SR. Activation of autophagy by alphaherpesviruses in myeloid cells is mediated by cytoplasmic viral DNA through a mechanism dependent on stimulator of IFN genes. J Immunol. 2011;187:5268-76.

31. Gillis PA, Okagaki LH, Rice SA. Herpes simplex virus type 1 ICP27 induces p38 mitogen-activated protein kinase signaling and apoptosis in HeLa cells. J Virol. 2009;83:1767-77.

32. Jackson BR, Noerenberg M, Whitehouse A. A novel mechanism inducing genome instability in Kaposi's sarcoma-associated herpesvirus infected cells. PLoS Pathog. 2014;10:e1004098.

33. Aubert M, Blaho JA. Modulation of apoptosis during herpes simplex virus infection in human cells. Microbes Infect. 2001;3:859-66.

34. Aubert M, Chen Z, Lang R, Dang CH, Fowler C, Sloan DD, Jerome KR. The antiapoptotic herpes simplex virus glycoprotein J localizes to multiple cellular organelles and induces reactive oxygen species formation. J Virol. 2008:82:617-29.

35. Rutkowski AJ, Erhard F, L'Hernault A, Bonfert T, Schilhabel M, Crump C, Rosenstiel P, Efstathiou S, Zimmer R, Friedel CC, Dolken L. Widespread disruption of host transcription termination in HSV-1 infection. Nat Commun. 2015:6:7126.

36. Sun L, Zhang Z, Bailey TL, Perkins AC, Tallack MR, Xu Z, Liu H. Prediction of novel long non-coding RNAs based on RNA-Seq data of mouse KIf1 knockout study. BMC Bioinformatics. 2012;13:331.

37. Trapnell C, Pachter L, Salzberg SL. TopHat: discovering splice junctions with RNA-Seq. Bioinformatics. 2009:25:1105-11.

38. Trapnell C, Williams BA, Pertea G, Mortazavi A, Kwan G, van Baren MJ, Salzberg SL, Wold BJ, Pachter L. Transcript assembly and quantification by RNA-Seq reveals unannotated transcripts and isoform switching during cell differentiation. Nat Biotechnol. 2010;28:511-5.

39. Sun L, Luo HT, Bu DC, Zhao GG, Yu KT, Zhang CH, Liu YN, Chen RS, Zhao Y. Utilizing sequence intrinsic composition to classify protein-coding and long non-coding transcripts. Nucleic Acids Res. 2013;41:e166.
40. Guttman M, Garber M, Levin JZ, Donaghey J, Robinson J, Adiconis X, Fan L, Koziol MJ, Gnirke A, Nusbaum C. Ab initio reconstruction of cell typespecific transcriptomes in mouse reveals the conserved multi-exonic structure of lincRNAs. Nat Biotechnol. 2010;28:503-10.

41. Pauli A, Valen E, Lin MF, Garber M, Vastenhouw NL, Levin JZ, Fan L, Sandelin A, Rinn JL, Regev A. Systematic identification of long noncoding RNAs expressed during zebrafish embryogenesis. Genome Res. 2012;22:577-91.

42. Barzilai A, Zivony-Elbom I, Sarid R, Noah E, Frenkel N. The herpes simplex virus type 1 vhs-UL41 gene secures viral replication by temporarily evading apoptotic cellular response to infection: Vhs-UL41 activity might require interactions with elements of cellular mRNA degradation machinery. J Virol. 2006;80:505-13

43. Esclatine A, Taddeo B, Evans L, Roizman B. The herpes simplex virus 1 UL41 gene-dependent destabilization of cellular RNAs is selective and may be sequence-specific. Proc Natl Acad Sci U S A. 2004;101:3603-8.

44. Esclatine A, Taddeo B, Roizman B. The UL41 protein of herpes simplex virus mediates selective stabilization or degradation of cellular mRNAs. Proc Natl Acad Sci U S A. 2004;101:18165-70.

45. Taddeo B, Zhang W, Roizman B. The UL41 protein of herpes simplex virus 1 degrades RNA by endonucleolytic cleavage in absence of other cellular or viral proteins. Proc Natl Acad Sci U S A. 2006;103:2827-32.

46. Taddeo B, Zhang W, Roizman B. The herpes simplex virus host shutoff RNase degrades cellular and viral mRNAs made before infection but not viral mRNA made after infection. J Virol. 2013;87:4516-22.

47. Trapnell C, Hendrickson DG, Sauvageau M, Goff L, Rinn JL, Pachter L. Differential analysis of gene regulation at transcript resolution with RNA-seq Nat Biotechnol. 2013;31:46-53.

48. Casero D, Sandoval S, Seet CS, Scholes J, Zhu YH, Ha VL, Luong A, Parekh C, Crooks GM. Long non-coding RNA profiling of human lymphoid progenitor cells reveals transcriptional divergence of B cell and T cell lineages. Nat Immunol. 2015;16:1282-91.

49. da Huang W, Sherman BT, Lempicki RA. Systematic and integrative analysis of large gene lists using DAVID bioinformatics resources. Nat Protoc. 2009;4:44-57.

50. Casaccia-Bonnefil P, Gu CH, Khursigara G, Chao MV. p75 neurotrophin receptor as a modulator of survival and death decisions. Microsc Res Tech. 1999:45:217-24

51. Tang B, Di Lena P, Schaffer L, Head SR, Baldi P, Thomas EA. Genome-wide identification of $\mathrm{BCl} 11 \mathrm{~b}$ gene targets reveals role in brain-derived Neurotrophic factor signaling. Plos One. 2011;6:e23691.

52. Lilley CE, Chaurushiya MS, Boutell C, Landry S, Suh J, Panier S, Everett RD, Stewart GS, Durocher D, Weitzman MD. A viral E3 ligase targets RNF8 and RNF168 to control histone ubiquitination and DNA damage responses. EMBO J. 2010;29:943-55.

53. Ferenczy MW, Ranayhossaini DJ, DeLuca NA. Activities of ICPO involved in the reversal of silencing of quiescent herpes simplex virus 1. J Virol. 2011;85: 4993-5002.

54. Roizman B. The checkpoints of viral gene expression in productive and latent infection: the role of the HDAC/COREST/LSD1/REST repressor complex. J Virol. 2011;85:7474-82.

55. Zhang L, Liu JJ, Li MT. Herpes simplex virus type 1 encephalitis and unusual retinitis in a patient with systemic lupus erythematosus. Lupus. 2013;22:1403-8.

56. Ernst J, Bar-Joseph Z. STEM: a tool for the analysis of short time series gene expression data. BMC Bioinformatics. 2006;7:191.

57. Madsen B, Spencer-Dene B, Poulsom R, Hall D, Lu PJ, Scott K, Shaw AT, Burchell JM, Freemont P, Taylor-Papadimitriou J. Characterisation and developmental expression of mouse Plu-1, a homologue of a human nuclear protein (PLU-1) which is specifically up-regulated in breast cancer. Mech Dev. 2002;119:S239-46.

58. Chiesa N, De Crescenzo A, Mishra K, Perone L, Carella M, Palumbo O, Mussa A, Sparago A, Cerrato F, Russo S, et al. The KCNQ1OT1 imprinting control region and non-coding RNA: new properties derived from the study of beckwith-wiedemann syndrome and silver-russell syndrome cases. Hum Mol Genet. 2012;21:10-25.

59. Fogarty S, Hardie DG. Development of protein kinase activators: AMPK as a target in metabolic disorders and cancer. Biochimica Et Biophysica ActaProteins Proteomics. 2010;1804:581-91.

60. Melis D, Pivonello R, Parenti G, Della Casa R, Salerno M, Balivo F, Piccolo P, Di Somma C, Colao A, Andria G. The growth hormone-insulin-like growth factor axis in glycogen storage disease type 1: evidence of different growth patterns and insulin-like growth factor levels in patients with glycogen storage disease type 1a and 1b. J Pediatr. 2010;156:663-U198. 
61. Woyach JA, Johnson AJ, Byrd JC. The B-cell receptor signaling pathway as a therapeutic target in CLL. Blood. 2012;120:1175-84

62. Fu C, Turck CW, Kurosaki T, Chan AC. BLNK: a central linker protein in B cell activation. Immunity. 1998:9:93-103.

63. Takeda K, Akira S. TLR signaling pathways. Semin Immunol. 2004;16:3-9.

64. Mogensen TH. Pathogen recognition and inflammatory signaling in innate immune defenses. Clin Microbiol Rev. 2009;22:240-73.

65. McCartney SA, Colonna M. Viral sensors: diversity in pathogen recognition. Immunol Rev. 2009;227:87-94.

66. Swennen ELR, Ummels V, Buss I, Jaehde U, Bast A, Dagnelie PC. ATP sensitizes H460 lung carcinoma cells to cisplatin-induced apoptosis. Chem Biol Interact. 2010;184:338-45.

67. Meng QJ, Lux A, Holloschi A, Li J, Hughes JMX, Foerg T, McCarthy JEG Heagerty AM, Kioschis P, Hafner M, Garland JM. Identification of Tctex2 beta, a novel dynein light chain family member that interacts with different transforming growth factor-beta receptors. J Biol Chem. 2006;281:37069-80.

68. Anderson KE, Marshall FJ. Behavioral symptoms associated with Huntington's disease. Adv Neurol. 2005;96:197-208.

69. Buckley NJ, Johnson R, Zuccato C, Bithell A, Cattaneo E. The role of REST in transcriptional and epigenetic dysregulation in Huntington's disease. Neurobiol Dis. 2010;39:28-39.

70. Johnson R, Buckley NJ. Gene dysregulation in Huntington's disease: REST, MicroRNAs and beyond. Neruomol Med. 2009;11:183-99.

71. Otsuki K, Uchida S, Wakabayashi Y, Matsubara T, Hobara T, Funato H, Watanabe Y. Aberrant REST-mediated transcriptional regulation in major depressive disorder. J Psychiatr Res. 2010;44:378-84.

72. Shulman JM, De Jager PL, Feany MB. Parkinson's disease: genetics and pathogenesis. Annu Rev Pathol. 2011;6:193-222.

73. Maraganore DM, Lesnick TG, Elbaz A, Chartier-Harlin MC, Gasser T, Kruger R, Hattori N, Mellick GD, Quattrone A, Satoh J, et al. UCHL1 is a Parkinson's disease susceptibility gene. Ann Neurol. 2004;55:512-21.

74. Hu B, Li X, Huo Y, Yu Y, Zhang Q, Chen G, Zhang Y, Fraser NW, Wu D, Zhou J. Cellular responses to HSV-1 infection are linked to specific types of alterations in the host transcriptome. Sci Rep. 2016;6:28075.

75. Pasieka TJ, Cilloniz C, Lu B, Teal TH, Proll SC, Katze MG, Leib DA. Host responses to wild-type and attenuated herpes simplex virus infection in the absence of Stat1. J Virol. 2009;83:2075-87.

76. Gong JP, Wang JJ, Ren K, Liu CA, Li B, Shi YJ. Serine/threonine kinase Pim-2 promotes liver tumorigenesis induction through mediating survival and preventing apoptosis of liver cell. J Surg Res. 2009;153:17-22.

\section{Submit your next manuscript to BioMed Central and we will help you at every step:}

- We accept pre-submission inquiries

- Our selector tool helps you to find the most relevant journal

- We provide round the clock customer support

- Convenient online submission

- Thorough peer review

- Inclusion in PubMed and all major indexing services

- Maximum visibility for your research

Submit your manuscript at www.biomedcentral.com/submit 